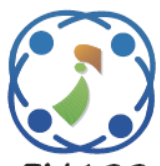

\title{
Analytical Study on IEEE 802.11ah Standard Impact of Hidden Node
}

\author{
Doan Perdana $^{1 *} \quad$ Sarah Hafidzah $^{1} \quad$ Bayu Erfianto $^{2}$ \\ ${ }^{1}$ Department Telecommunication Engineering, Faculty of Electrical Engineering, \\ Telkom University, Bandung, West Java, Indonesia \\ ${ }^{2}$ Department Technical Information, Faculty of Informatics, Telkom University, Bandung, West Java, Indonesia \\ * Corresponding author's Email: doanperdana@telkomuniversity.ac.id
}

\begin{abstract}
The IEEE 802.11ah standard was developed from IEEE 802.11, which works on a Wireless Local Area Networks (WLAN). This standard works in the sub-band $1 \mathrm{GHz}$, to increase the range of an Access Point (AP) up to 1 $\mathrm{km} 2$, with the ability to handle approximately 8000 stations (STA). The IEEE 802.11ah standard occurs in the MAC and PHY layers. However, the high number of STAs produces high collision, therefore this standard introduces the Restricted Access Window (RAW) at the MAC layer. This research accurately examines a surrogate model to predict RAW performance under hidden nodes scenario. The solution to the surrogate model was analyzed using the Markov chain and numerically simulated with Matlab. Furthermore, the Markov chain solution is used to determine the performance measure parameters, namely throughput, energy consumption, and average delay. This research also examines the effect of hidden nodes on the IEEE 802.11 ah standard network's performance, especially in RAW, with variables obtained using Bianchi's approach. The result showed that the simulated RAW slot duration of $52 \mu \mathrm{s}$ on the IEEE 802.11ah standard made performance results better than $104 \mu \mathrm{s}$ and $156 \mu \mathrm{s}$. The effect of the hidden nodes makes the successful transmission time probability longer due to its increment.
\end{abstract}

Keywords: IEEE 802.11ah, WLAN, Matlab, RAW, Bianchi, Hidden node.

\section{Introduction}

Wireless Fidelity (Wi-Fi) is a generic term that was initially designed to provide wireless internet services to several devices, such as computers, mobile phones, and other equipment (printers and video cameras) at an extremely high speed. 802.11 ah (Wi-Fi Ha-Low) is a Wireless Local Area Network (WLAN) specification developed by the IEEE (Institute of Electrical and Electronics Engineers) that operates in frequency bands below one gigahertz $(900 \mathrm{MHz})$ while delivering a range nearly twice that of the previous Wi-Fi technologies. It increases the reach of an Access Point (AP) to $1 \mathrm{~km} 2$ and controls relatively 8000 stations (STA). However, the height of the STA increases collision probability [1].

Wi-Fi Ha-Low introduces a Restricted Access Window (RAW), which divides stations into different groups, and only allows those in a particular group to access the channel simultaneously, in order to reduce collisions probability in crowded networks, thereby achieving better performance [2, 3]. RAW slots reduce energy consumption by entering the sleep mode when the station is not in use [4]. However, it only grants some nodes access to channels with in a certain period, although there is a possibility of hidden node collision in the same RAW, which impacts energy efficiency [5].

In a previous research about the effect of IEEE 802.11ah standard mobility with a traffic pattern change scheme [6], it had not yet discussed the mathematical model approach so that in this research, a mathematical model using the Bianchi approach. The Bianchi approach calculates collisions probability in RAW without calculating the limited length of RAW slots [7, 8].

In this research, the author examines the IEEE 802.11ah standard through the Bianchi approach to reduce collisions due to hidden nodes.

The IEEE 802.11ah standard has many STAs in one AP, so it is difficult to find a mechanism that can 
reduce collisions on network traffic. A collision occurs because two or more STAs try to access a channel simultaneously, known as Multiple Access. Multiple access occurs because each node fails to recognize the other nodes or node is not within the scope of another STA's area.

One of the problems with multiple access is the hidden node problem, to avoid hidden nodes by adding the RTS / CTS mechanism and the RAW mechanism. RTS / CTS is a handshake protocol for sending and receiving frames, which is a mechanism of Distributed Coordination Function (DCF)[9].

The effect of IEEE 802.11 ah standard mobility on changing traffic patterns is reported in this research [10]. However, the mathematical analysis for formalizing the results was not discussed. Although the IEEE 802.11ah standard has numerous STAs in one AP, it is difficult to discover a mechanism that reduces network traffic collisions. A collision usually occurs when two or more STAs try to access a channel simultaneously, and this is referred to as Multiple Access [11, 12]. It also occurs when nodes fail to recognize one another or are not within the scope of STA [13].

A certain problem associated with multiple accesses is the issue of the hidden node, although this is avoided by adopting a common method that involves combining the RTS or CTS and RAW mechanisms [14]. RTS or CTS is a handshake protocol for sending and receiving frames, a mechanism of Distributed Coordination Function (DCF) $[15,16]$.

This research adopts the Markov chain model and the Bianchi approach initially designed for the IEEE 802.11 standard. This approach is used to predict the collision probability using Matlab [17]. However, it cannot determine the limited length of the RAW slots $[18,19]$. Therefore, certain parameters were included in the Bianchi Markov chain solution to examine the IEEE 802.11 ah standard in reducing collisions due to hidden nodes.

\section{Related works}

The IEEE 802.11ah standard has a Physical (PHY) and Medium Access Control (MAC) protocol layer that operates on a $1 \mathrm{GHz}$ sub-band frequency with data rates between $150 \mathrm{kbps}$ and $8 \mathrm{Mbps}$ as well as the Restricted Access Window (RAW) mechanism. The Markov chain model in WLAN adopted the Bianchi approach [20] to calculate the saturated throughput of IEEE 802.11 DCF, under the assumptions of having error-free channels and unlimited packet retransmission. S. G. Yoon, J. O. Seo, S. Bahk and Liangxiao Xin, D. Starobinski. developed. a Bianchi model and a collision probability theory, respectively, based on the hidden nodes [21, 22].

The 802.11ah standard derived its characteristics from $802.11 \mathrm{ac}$, and it was further adjusted to $1 \mathrm{GHz}$ sub-band frequency. Furthermore, its channel bandwidth ranges from $1 \mathrm{MHz}$ to $16 \mathrm{MHz}$. When it is operated at a low frequency and narrow bandwidth, it causes it to transmit over a long-range (approximately $1 \mathrm{~km}$ ) with its power consumption less than the conventional Wi-Fi technology that uses $2.4 \mathrm{GHz}$ and $5 \mathrm{GHz}$ sub-band frequencies [23]. However that is produces high collision, therefore this standard introduces the Restricted Access Window (RAW) at the MAC layer.

At the PHY layer, IEEE 802.11ah supports multiple transmission data rates represented by the Modulation and Coding Scheme (MCS). The stations are allowed to select the MCS dynamically, thereby adapting to the wireless channel [19]. The MAC layer in the 802.11ah standard introduces the Restricted Access Window (RAW) mechanism, which supports the station's deployment to conserve energy [23]. Certain research regarding the MAC layer's optimization mechanisms for energy and throughput analysis was reviewed $[4,24,25]$. Previous studies based on RAW mechanism model on MAC for the IEEE 802.11ah standard were also discussed [2, 19].

The RAW mechanism aims to reduce collision probability and energy consumption by dividing all STAs into groups and setting time intervals referred to as RAW slots as well as allocating each group to them $[26,27]$. The distribution of STAs to access channels is also regulated using a duration set by the slot. The STA in the same group competes for channel access using the DCF procedure while the back-off procedure uses Enhanced Distribution Channel Access (EDCA) to process transmissions both inside and outside each RAW slot [4, 28, 29].

Fig. 1 shows that AP 802.11ah determines the time interval called RAW of duration $\sigma$ and sets each on the STA list to reduce contention. However, only registered STAs can access the channel, with the following equation used to determine the length of duration in RAW $(\sigma)[19,28]$ :

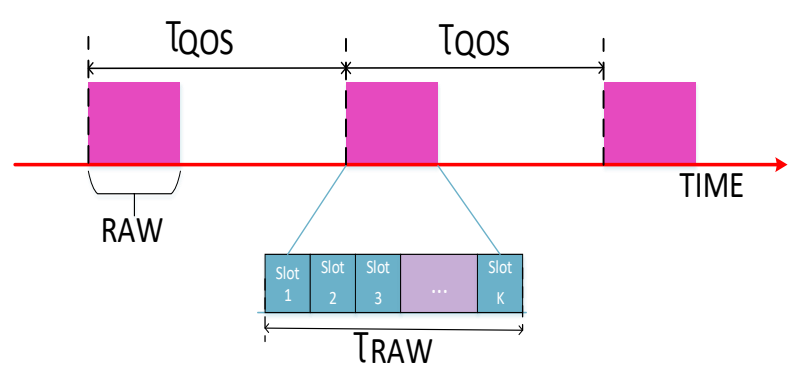

Figure. 1 Restricted access window (RAW) [1] 


$$
\sigma=500 s \mu s+C \cdot 120 \mu s
$$

The $\mathrm{C}$ in the equation denoted the packet length consequently, AP schedules the Resource Allocation frame as the first to be transmitted at the beginning of the RAW, including the channel access rules [19]. The beginning of the RAW is further defined as the start time of its assignment subfield and the RAW parameter set (RPS) element [30].

\section{Research method}

The design of this research illustrates the overall system of blocks in Fig. 2.

Firstly, the IEEE 802.11ah RAW standard is determined in this research. Secondly, mathematical calculations were divided into two, the normal and the hidden node affected conditions. The data from these calculations were used to determine the delay, throughput, and energy consumption.

\subsection{Markov chain model}

The Bianchi approach was originally established for conventional Wi-Fi. This method is a form of the Markov Chain approach designed for the IEEE 802.11 standard. This analysis is a type of probability theory, commonly known as stochastic models used to model randomly changing systems. It is assumed that future conditions depend only on the present state and not on the sequence of preceding events (assuming Markov properties) [31].

Bianchi introduces virtual slots, namely the time interval between two consistent changes to the backoff counter. The mathematical model is used to develop the assumption that each STA has a frame that needs to be transmitted, and the process is stable. Therefore, this process is carried out in a particular virtual slot that does not change over time [1]. Arbitration Inter Frame Space (AIDS) is used in the

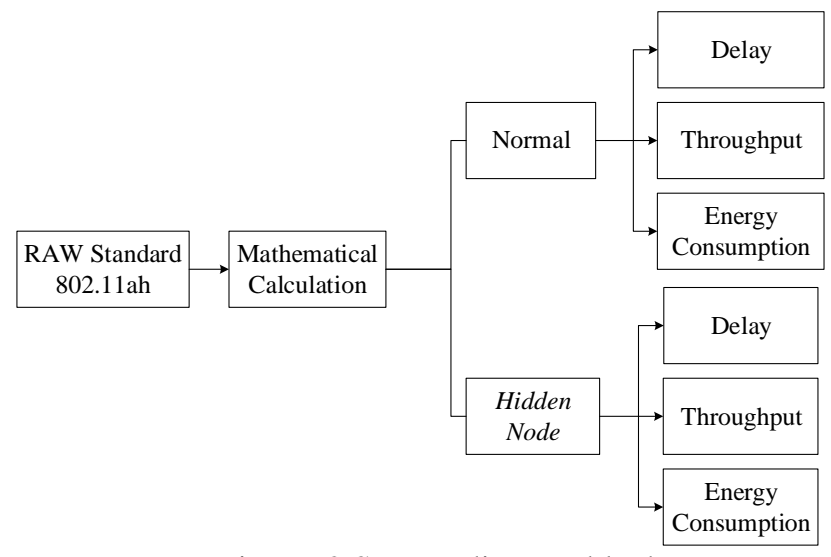

Figure. 2 System diagram blocks

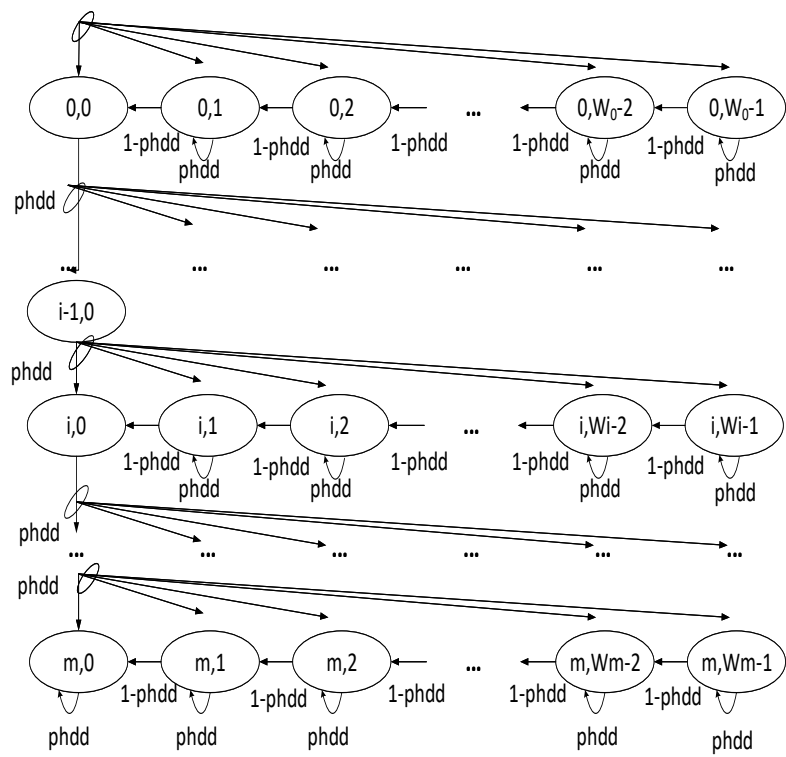

Figure. 3 Markov chain IEEE 802.11ah in hidden nodes condition

Bianchi approach. It is a standard version of DCF Inter Frame Space DIFS from its mechanism.

The Markov Chain model is shown in Fig. 3, and it is evident that all nodes have access to the channel, although when it is busy, a collision tends to occur. The system duplicates the contention window in addition the new back-off time $W=C W m i n+1$ is calculated $[32,33]$.

The value of $i$ is the back-off value where $0 \leq i \leq m$, assuming the transmission is unsuccessful, the backoff stage $(m)$ is reset, supposing the reverse is the case, with the back-off restarted in order to send a new packet [34]. The movement of one state to another when the channel is busy occurs at a probability of 1phdd.

The mathematical model used is divided into two, during the normal conditions and when influenced by hidden nodes [21, 35]. However, under normal conditions, the collision probability ( $\mathrm{p}$ ) is formulated by the following equation $[22,33]$.

$$
p=1-e^{(-\rho i)}
$$

The traffic load on the node is denoted by $\rho i=\lambda$ $T$ where the value of $\lambda, 8,125$ packets / second and $T$ the transmission duration of each packet of 0.016 seconds is obtained by adopting the results of the research [22]. While under conditions affected by hidden nodes, collision probability $(P h d d)$ is formulated by Eq. (3) [22].

$$
\text { Phdd }=1-e^{(-\rho i)}(1-\rho i)
$$


The probability of a node to transmit in a randomly selected time slot is defined as $\tau$, and it is formulated in Eq. (4). However, the Markov chain solution is shown in Fig. 3 [36].

$\tau=\frac{2(1-2 p)}{(1-2 p)(C W \min +1)+p C W \min \left(1-(2 p)^{m}\right.}$

Where the value of $C W \min$ is the contention window size and $m$ is the maximum back-off stage using the collision probability value $(p)$ formulated in Eq. (2) for normal conditions and Eq. (3) for hidden nodes. Immediately the value $\tau$ is realized, Eq. (5) and (6) which proposes that the probability of one node transmits a packet in the time slot (Ptr), and the packet delivery is successful $(P s)$ with $\mathrm{n}$ values (where $\mathrm{n}$ is the number of nodes) was therefore proposed.

$$
\begin{gathered}
\text { Ptr }=1-(1-\tau) \\
P s=\frac{n \tau(1-\tau)^{n-1}}{P t r}
\end{gathered}
$$

Although, under normal condition, the values of $T s$ and $T c$ serves as the duration of the successful packet and collision packet respectively, as shown in Eq.s (7) and (8) [37]. To reduce the occurrence of collisions under the hidden node condition, the RTS or CTS mechanism was applied to obtain the successful packet duration, which was determined $T s_{R T S / C T S}$ as shown in Eq. (9) while $T c_{R T S / C T S}$ [38]. However, this does not change the transition probability in the Markov chain.

$$
\begin{gathered}
T s=A I F S+T \text { Data }+ \text { TACK } \\
+T P H Y \\
T c=A I F S+\text { TData }+T P H Y
\end{gathered}
$$

$$
\begin{aligned}
\text { TSRT S/CT S } & \\
& =\text { TS + TRT S }+3 \\
& * \text { SIFS }
\end{aligned}
$$

The adopted parameters are listed in Table 1, and to validate the solution, numerical simulations that define two scenarios [1,38]. The first stated that under normal conditions, the RAW slot duration parameter and the number of Nodes are changed twice and thrice. Consequently, out of all the 30 nodes initiated, the normal ones were determined
Table 1. Table caption

\begin{tabular}{|l|l|}
\hline Parameters & Value \\
\hline $\begin{array}{l}\text { Physical } \\
\text { Layer }\end{array}$ & WLAN/IEEE \\
Transport Layer & UDP \\
\hline Payload & 8184 bit \\
\hline Total STA & 30 \\
\hline $\mathrm{m}$ & 6 \\
\hline RAW slot & 1 \\
\hline$\lambda$ & 8125 packet/second \\
\hline T & $0,016 \mu \mathrm{s}$ \\
\hline$\tau$ S & $1064 \mu \mathrm{s}$ \\
\hline$\tau$ C & $1064 \mu \mathrm{s}$ \\
\hline$\tau$ e & $52 \mu \mathrm{s}$ \\
\hline TDATA & $348 \mu \mathrm{s}$ \\
\hline Tsym & $40 \mu \mathrm{s}$ \\
\hline TPHY & $6 \times \mathrm{Tsym}$ \\
\hline TACK & $6 \times \mathrm{Tsym}$ \\
\hline TRTS & $122+(6 \mathrm{x}$ Tsym $)$ \\
\hline TCTS & $6 \times \mathrm{Tsym}$ \\
\hline CWmin & 32 \\
\hline CWmax & 1023 \\
\hline SIFS & $160 \mu \mathrm{s}$ \\
\hline AIFS & SIFS $+(3 \times \tau$ e) \\
\hline Transmit $(\mathrm{Pt})$ & $250 \mathrm{~mW}$ \\
\hline Receive $($ Prx $)$ & $135 \mathrm{~mW}$ \\
\hline Idle $($ Pi $)$ & $1.5 \mathrm{~mW}$ \\
\hline & \\
\hline
\end{tabular}

randomly at an AP range of $1000 \mathrm{~m}$. This scenario utilizes the Bianchi approach to calculate performances in the form of delay, throughput, and energy consumption.

According to the second scenario, the condition of a channel affected by a hidden node changes the RAW slot duration parameter and the number of Nodes twice or thrice. Subsequently, out of all the 30 initiated nodes, hidden nodes were randomly determined with approximately $1000 \mathrm{~m}$ outside the AP scope. This scenario adopted the Bianchi approach to account for the delay, throughput, and energy consumption.

\section{Result and discussion}

Based on the numerical simulation of two scenarios, the results are obtained in the metrics of delay, throughput, and energy consumption and are stated as follows:

According to Fig. 4, a circle shows the AP range limit at a distance of $1000 \mathrm{~m}$. Furthermore, out of all the 30 nodes initiated, the normal ones were determined randomly at an AP range of $1000 \mathrm{~m}$. The 


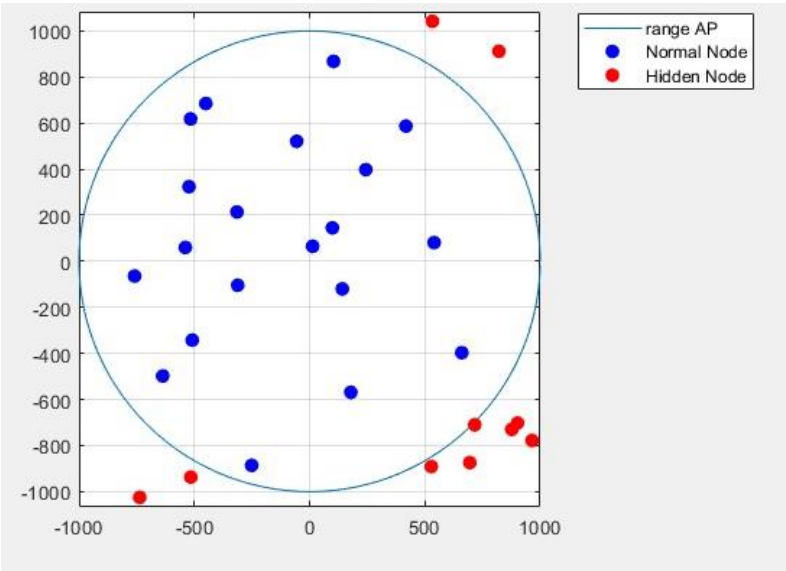

Figure. 4 Nodes

normal (20 in number) and hidden (10) nodes are represented in blue and red, respectively.

\subsection{Delay}

The values of delay are calculated after Ptr and $P S$ have been obtained using Eq. (10). Ts and $T c$ are the successful packet and collision packet durations respectively, and $\sigma$ is the slot duration for normal conditions [38].

$$
=\frac{\left(1-P_{t r}\right) \sigma+P_{t r}\left(1-P_{s}\right) \times T_{C}+P_{t r} x P_{s} \times T_{s}}{P_{t r} x P_{s}}
$$

Fig. 5 and Fig. 6 shows the duration of RAW slot $52 \mu \mathrm{s}$ which was adopted in this research, is better than $104 \mu \mathrm{s}$ and $156 \mu \mathrm{s}$ [33]. This is because the RAW slot duration is directly proportional to the delay value, as shown in Eq. (10), and a decrease in the value of the duration causes a reduction in delay. The duration of the RAW slot is stated in Eq. (1). An increase in the extended packet $(C)$ tends to extend

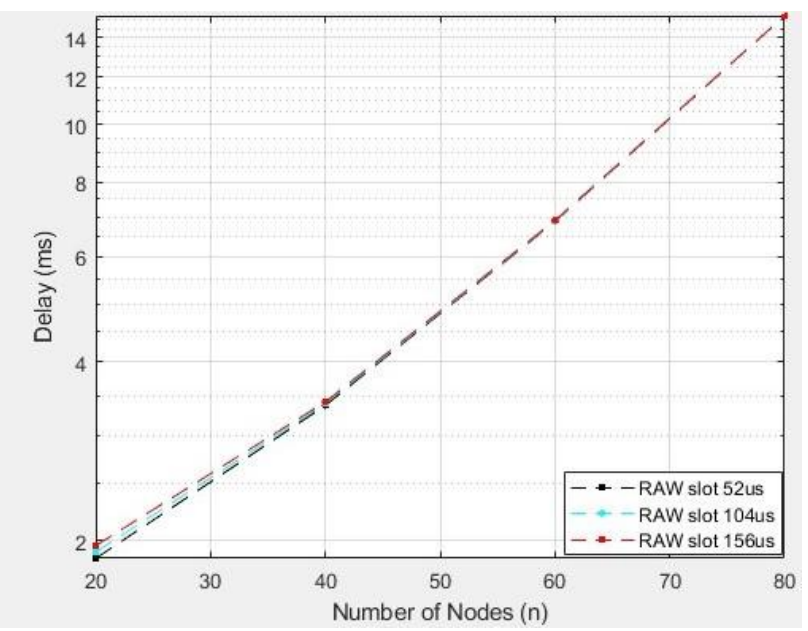

Figure. 5 Average delay of normal nodes

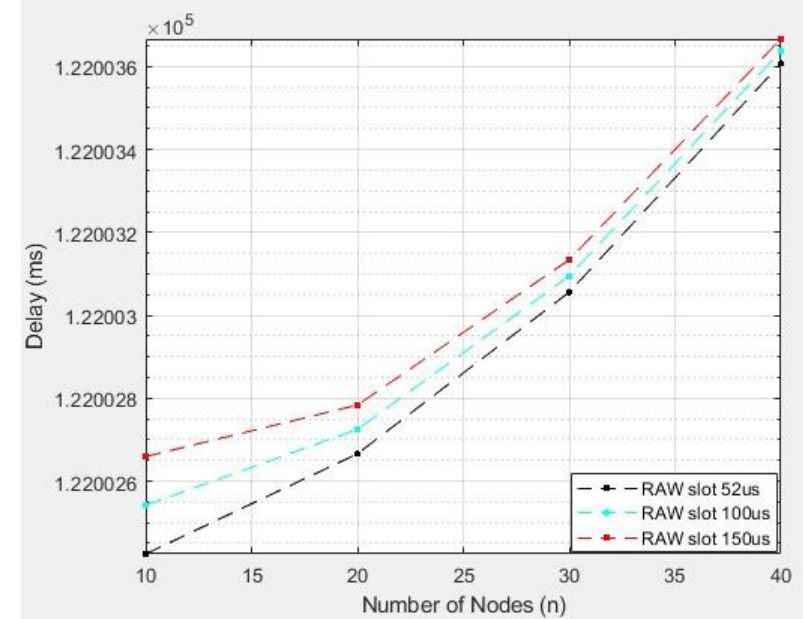

Figure. 6 Average delay of hidden nodes

the duration of RAW,resulting in more delay, reduced throughput, and the amount of energy consumed.

Delay in normal condition is reducing, unlike in the hidden node state, as shown in Fig. 5 and 6. The duration of success transmission $(T s)$ in normal conditions is lower than the state affected by the hidden node. The RTS/CTS mechanisms in the hidden state make the duration of success transmission $(T S)$ and delay longer.

\subsection{Throughput}

Throughput is defined as the rate of effective data transfer, and it is measured in bytes per second (Bps), which is equivalent to the total number of packets received in bits divided by the amount of time sent [39]. It is determined by the following equation. [38]:

$$
\begin{aligned}
& \text { Throughput } \\
& =\frac{P_{S} P_{t r} \text { lpayload }}{\left(1-P_{t r}\right) \sigma+P_{t r} P_{s} T_{s}+P_{t r}\left(1-P_{S}\right) T_{c}}
\end{aligned}
$$

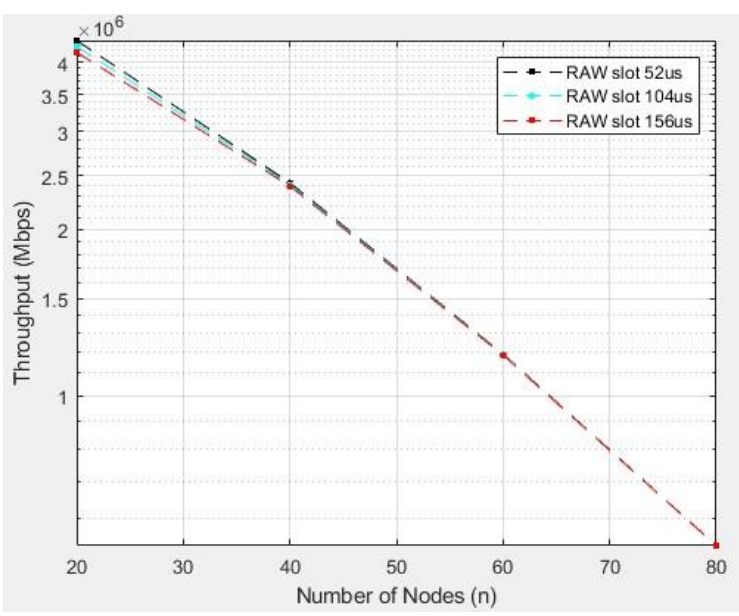

Figure. 7 Average throughput of normal nodes 


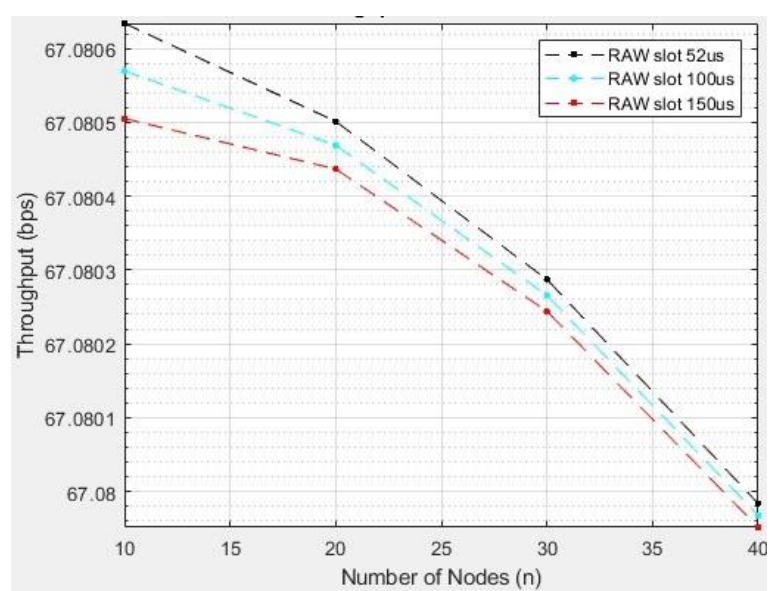

Figure. 8 Average throughput of hidden nodes

Fig. 7 and Fig. 8 shows the duration of RAW slot $52 \mu \mathrm{s}$ in this research [33], which is determined to be better than $\mu \mathrm{s}$ and $156 \mu \mathrm{s}$. This is because RAW slot duration is inversely proportional to the throughput value, as shown in Eq. (11). However, a decrease in its value causes an increase in throughput. The duration of the RAW slot is stated in Eq. (1). Therefore, an increase in the extended packet $(C)$ extends the RAW duration, which increases delay and reduces throughput, including the amount of energy consumed.

Throughput under normal conditions (Fig. 7) is higher than the state affected by hidden nodes (Fig. $8)$. This is because the probability of collision $(p)$ in the normal condition is smaller, however, it is greater in the probability of transmission (Ptr), thereby causing an increase in the throughput. The RTS/ CTS mechanism on the hidden node reduces collision. Therefore, the successful delivery time $T s_{R T S / C T S}$ tends to be longer while the throughput is smaller.

\subsection{Energy consumption}

Equation [33] is used to determine the energy consumption when transmitting packet data, and it is stated as follows:

$$
e=\frac{\left(1-P_{t r}\right) \sigma+P_{t r} P_{s} T_{s}+P_{t r}\left(1-P_{s}\right) T_{c}}{P_{S} P_{t r} \text { payload }}
$$

$E c$ and $E s$ are the average energy needed for the collision and success transmission, respectively, as stated in Eq. (13) and (14). This is similar to RTS/CTS stated in equation $[38,39]$.

$$
\begin{gathered}
E s=(A I F S \cdot P i)+\left(T_{D A T A} \cdot P_{t x}\right) \\
+\left(T_{P H Y} \cdot P i\right)
\end{gathered}
$$

$$
\begin{aligned}
E S=(A I F S \cdot & P i)+\left(T_{D A T A} \cdot P_{t x}\right) \\
& +\left(T_{P H Y} \cdot P i\right) \\
& +\left(T_{A C K} \cdot P_{r x}\right) \\
E_{\frac{S_{R T S}}{T T S}}=E S+ & \left(T_{R T S} \cdot P_{t x}\right) \\
& +\left(T_{C T S} \cdot P_{t x}\right) \\
& +\left(3 \cdot S I F S \cdot P_{i}\right)
\end{aligned}
$$

Fig. 9 and Fig. 10 show the slot RAW duration is $52 \mu$ s adopted in this research is better than $104 \mu \mathrm{s}$ and $156 \mu$ s [38]. This is because the RAW slot duration is directly proportional to energy consumption, as stated in Eq. (12). Furthermore, a decrease in the value of RAW duration also causes a decrease in energy consumption.

The RAW slot duration is stated in Eq. (1), and an increase in the extended packet $(C)$ causes an increase in the extended duration of RAW, which results in more delay, reduced throughput, including the amount of energy consumed. Energy

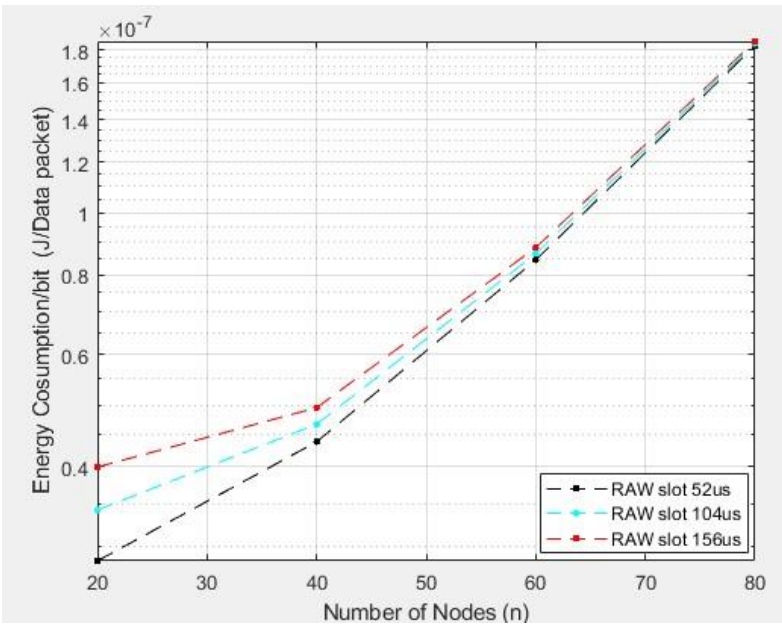

Figure. 9 Average energy consumption of normal nodes

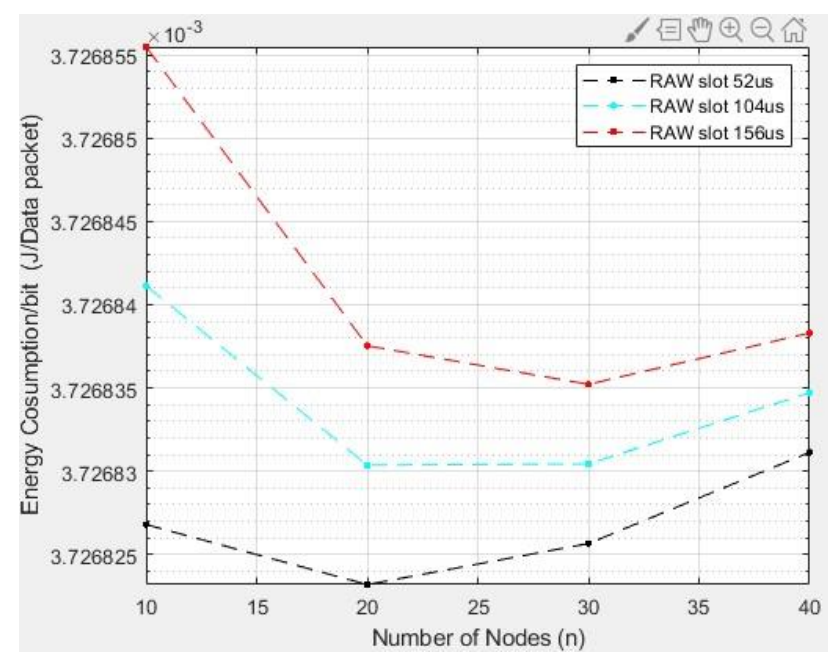

Figure. 10 Average energy consumption of hidden 
consumption in normal conditions is less than the delay in a state affected by hidden nodes. This is because the duration of success $(T s)$ in Eq. (7) under normal conditions tends to decrease when in a state of hidden nodes $E S_{\text {ssTicrs }}$ in Eq. (9). The hidden node condition results in a higher probability of collision, therefore an RTS or CTS mechanism needs to be included in order to increase the duration of success. As a result, it requires a longer time, thereby increasing the value of delay, and energy consumption.

Energy consumption is also influenced by the probability of transmission (Ptr). When several nodes are sent, the transmission probability is higher as well as energy consumption. According to Eq. (5), Ptr's value is also influenced by the probability of a node trying to transmit $(\tau)$. The value of $\tau$ tends to be high when the probability of collision $(p)$ is low, which results in a higher Ptr. The value of the probability of success $(P s)$ is inversely proportional to Ptr, as stated in Eq. (6). Energy consumption at IEEE standard $802.11 \mathrm{ah}$ is less with an average of $3.72682 \mathrm{~mJ} /$ data compared to IEEE standard 802.11e [40].

\section{Conclusion}

This research analyses the effect of RAW and hidden nodes on the 802.11ah standard using the Bianchi approach. The metrics or performance measures of interests are throughput, delay, and energy consumption. Based on the simulation, it is discovered that the IEEE 802.11ah standard with $52 \mu \mathrm{s}$ RAW slot duration is better than $104 \mu \mathrm{s}$ and $156 \mu \mathrm{s}$. The smaller the duration value, the more effective is the performance results. An increase in the extended packet $(C)$ causes an increase in RAW duration, which results in greater delay, reduced throughput, and the amount of energy consumed. One of the problems with multiple access is the hidden node problem, to avoid hidden nodes by adding the RTS / CTS mechanism and the RAW mechanism. The RTS or CTS mechanism on the hidden node reduces collision, and the successful delivery time $\left(T s_{R T S / C T S}\right)$ tends to be longer under normal conditions, thereby causing an increase in delay $122.00293 \mathrm{~s}$ and energy consumption $3.72682 \mathrm{~mJ} /$ data packet. The more nodes are sent, the higher the transmission probability (Ptr). Ptr's value is also influenced by the probability of a node trying to transmit $(\tau)$. The value of $\tau$ tends to be high when the probability of collision $(p)$ is low, which results in an increased Ptr. The value of the probability of success $(P S)$ is inversely proportional to the value of Ptr.

\section{Conflicts of Interest}

The authors declare that they have no conflict of interest, and no personal circumstances or interest that may be perceived as inappropriately influencing the representation or interpretation of reported research results.

\section{Author Contributions}

This research article consists of several authors, Doan Perdana and Sarah Hafidzah for contributing to the completion of the main work, such as proposing the idea, design system, coding, testing, and writing the paper. And also, to Bayu Erfianto for carrying out the editing.

\section{Acknowledgments}

The authors are grateful to Telkom University that supported this funding research.

\section{References}

[1] E. Khowov, A. Krotov, A. Lyakhof, R. Yusupov, M. Condoluci, M. Dohler, and I. F. Akyildiz, "Enabling the iot with wi-fi halow performance evaluation of restricted access window", IEEE Access, Vol. 4, 2016.

[2] L. Tian, J. Famaey, and S. Latre, Evaluation of the IEEE 802.11ah Restricted Access Window mechanism for dense IoT networks, 2016.

[3] J. Kim and I. Yeom, "Qos enhanced channel access in ieee 802.11ah networks", In: Proc. of 17th Int. Symp. Commun. Inf. Technol. Isc., 2017.

[4] M. Qutab-Ud-Din, A. Hazmi, B. Badihi, A. Larmo, J. Torsner, and M. Valkama, "Performance analysis of iot-enabling ieee 802.11ah technology and its raw mechanism with non-cross slot boundary holding schemes", Proceedings of the WoWMoM 2015: A World of Wireless Mobile and Multimedia Networks, 2015.

[5] L. Zhang, H. Li, Z. Guo, L. Ding, F. Yang, and L. Qian, Signal strength assistant grouping for lower hidden node collision probability in 802.11ah, 2017.

[6] W. Choi, "Clustering Algorithm for Hidden Node Problem in Infrastructure Mode IEEE 802.11 Wireless LANs", In: Proc. of 2008 10th International Conference on Advanced Communication Technology, Gangwon, Korea (South), pp. 1335-1338, 2008, doi: 10.1109/ICACT.2008.4494011.

[7] J. Lee and I. Yeom, "Avoiding collision with hidden nodes in IEEE 802.11 wireless 
networks", IEEE Communications Letters, Vol. 13, No. 10, pp. 743-745, 2009, doi: 10.1109/LCOMM.2009.090975.

[8] L. B. Jiang and S. C. Liew, "Improving Throughput and Fairness by Reducing Exposed and Hidden Nodes in 802.11 Networks", in IEEE Transactions on Mobile Computing, Vol. 7, No. 1, pp. 34-49, 2008, doi: 10.1109/TMC.2007.1070.

[9] J. Jeong, H. Kim, S. Lee, and J. Shin, "An analysis of hidden node problem in IEEE 802.11 multihop networks", In: Proc. of the 6th International Conference on Networked Computing and Advanced Information Management, Seoul, Korea (South), pp. 282285, 2010.

[10] R. M. N. Ajinegoro, "Analisis Pengaruh Mobilitas pada Standard IEEE 802.11ah dengan Skema Perubahan Traffic Pattern", Telkom University, 2017.

[11] S. Gamage, J. Y. Khan, and D. T. Ngo, "Adaptive re- source allocation with traffic peak duration prediction and admission control for cognitive wi-fi networks", Computer Networks, Vol. 142, pp. 240-252, 2018. [Online]. Available:

https://www.sciencedirect.com/science/article/ pii/S1389128618 304122.

[12] T. V. P. Santhappan, S. Patro, B. R. Tamma, and A. A. Franklin, "Network coordination function for uplink traffic steering in tightly coupled lte wi-fi networks", Computer Networks, Vol. 127, pp. 296-316, 2017. [Online]. Available: https://www.sciencedirect.com/science/article/ pii/S1389128617 303420.

[13] J. Fu, Z. Zhou, L. Zheng, J. Wang, and X. Li, "Throughput analysis of IEEE 802.11 DCF in presence of hidden nodes", In: Proc. of 2011 International Conference on Multimedia Technology, Hangzhou, China, pp. 920-922, 2011, doi: 10.1109/ICMT.2011.6003011.

[14] C. Hu, "Approximation algorithms of minimizing hidden pairs in 802.1 1ah networks", IEEE Access, Vol. 7, p. 170742-170752, 2019.

[15] S. R. Inamdar, S. B. Basavaiah, and R. M. Yadahalli, "Co-Operative directional routing protocol for MANET", International Journal of Intelligent Engineering and Systems, Vol. 11, no. 2, pp. 93-101, 2018, doi: 10.22266/IJIES2018.0430.11.

[16] V. Banos-Gonzalez, M. S. Afaqui, E. LopezAguilera, and E. Garcia Villegas, "Ieee 802.11ah: A technology to face the iot challenge", IEEE Access, Vol. 7, p. 170742170752, 2016.
[17] S. Fan, J. Li, J. Li, and H. Sun, "Performance analysis of wireless network measurement control system using Matlab/Simulink", International Journal of Intelligent Engineering and Systems, Vol.. 3, No. 2, pp. 42-49, 2010, doi: 10.22266/ijies2010.0630.06.

[18] O. Raeesi, J. Pirskanen, A. Hazmi, T. Levanen, and M. Valkama, "Performance evaluation of ieee 802.11ah and its restricted ac- cess window mechanism", ser. IEEE International Conference on Communications Workshops, 2014, pp. 460-466, doi: 10.1109/ICCW.2014.6881241, 2014.

[19] L. Tian, M. Mehari, E. De Poorter, S. Latr, J. Famaey, and E. L. E. Garciavillegas, "Optimization-oriented raw modeling of ieee 802.11ah heterogeneous networks", IEEE Internet Things J., Vol. 4662, No. C, pp. 1-12, 2019.

[20] G. Bianchi, "Performance analysis of the ieee 802.11 distributed coordination function", IEEE Journal on Selected Areas in Communications, Vol. 18, No. 3, 2000.

[21] S. G. Yoon, J. O. Seo, and S. Bahk, "Regrouping algorithm to alle- viate the hidden node problem in 802.11ah networks", Computer Networks, Vol. 105, p. 22-32, 2016

[22] L. Xin and D. Starobinski, Mitigation of cascading denial of service attacks on $\mathrm{Wi}-\mathrm{Fi}$ networks, 2018.

[23] L. Tian, S. Latre, S. Deronne, and J. Famaey, "Implementation and validation of an ieee 802.11ah module for ns-3", In: ACM-ICN 2016 Proceedings of the 2016 3rd ACM Conference on Information Centric Networking, p. 249-254, 2016.

[24] P. K. Reddy and R. Babu, "An evolutionary secure energy efficient routing protocol in Internet of Things", International Journal of Intelligent Engineering and Systems, Vol.. 10, No. 3, pp. 337-346, 2017, doi: 10.22266/ijies2017.0630.38

[25] I. Iala, I. Dbibih, and O. Zytoune, "Adaptive duty-cycle scheme based on a new prediction mechanism for energy optimization over IEEE 802.15.4 Wireless Network", International Journal of Intelligent Engineering and Systems, Vol. 11, No. 5, pp. 105-113, 2018, doi: 10.22266/IJIES2018.1031.10.

[26] W. Yin, P. Hu, W. Wang, J. Wen, and H. Zhou, "Fasus: A fast association mechanism for 802.11ah networks", Computer Networks, Vol. 175, p. 107287, 2020. [Online]. Available: https://www.sciencedirect.com/science/article/ pii/S1389128619 312083 
[27] P. Campos, A. Hernandez-Solana, and A. Valdovi- nosBardaji, "Analysis of hidden node problem in lte networks deployed in unlicensed spectrum", Computer Networks, Vol. 177, p. 107280, 2020. [Online]. Available: https://www.sciencedirect.com/science/article/ pii/S1389128620 300396.

[28] A. Sljivo, D. Kerkhove, L.Tian, J. Famaey, A. Munteanu, I. Moerman, J. Hoebeke, and E. D. Poorter, "Performance evaluation of ieee 802.11ah networks with high-throughput bidirectional traffic", Sensors (Switzerland), Vol. 18, No. 2, 2018.

[29] B. Rajkumar and G. Narsimha, "Secure light weight encryption protocol for MANET", International Journal of Intelligent Engineering and Systems, Vol. 10, No. 3, pp. 58-65, 2017, doi: 10.22266/ijies2017.0630.07.

[30] C. I. Society, "Ieee standard for information technology - telecom- munications and information exchange between systems local and metropolitan area networks - specific requirements part 11: Wireless lan medium access control (mac) and physical layer (phy) specification, ieee std 802.11 $\mathrm{ah}^{\mathrm{TM}}-2016$ (amendment to ieee std $802.11^{\mathrm{TM}}-2016$ as amended by ieee std 802.11ai'TM-2016)", IEEE, 2016.

[31] Usu. Model markov, 2020. [Online]. Available: http://repository.usu.ac.id/bitstream/handle/123 456789/56510/ II.pdf? sequence $=3$ is Allowed $=\mathrm{y}$.

[32] A. Abu-Khadrah, Z. Zakaria, M. Othman, and M. S. I. Mohd Zin, "Using markov chain model to evaluate the performance of edca protocol under saturation and non-saturation conditions", Int. Rev. Comput. Softw., Vol. 10, No. 3, p. 315323, 2015.

[33] A. Abu-Khadrah, Z. Zakaria, M. Othman, and M. S. I. Mohd Zin, "Analytic modeling of the coexistence of ieee 802.15.4 and ieee 802.11 in saturation conditions", IEEE Commun. Lett., Vol. 19, No. 11, p. 1981-1984, 2015.

[34] S. Ray, D. Starobinski, and J. B. Carruthers, "Mathematical and simulation analysis of contention resolution mechanism for ieee 802.11ah networks", Computer Communications, Vol. 28, No. 10, pp. 1179$1192,2005$.

[35] Z. Wu and Y. H. Hu, "How many wireless resources are needed to resolve the hidden terminal problem?", Computer Networks, Vol. 57, No. 18, pp. 3987-3996, 2013. [Online]. Available: https://www.sciencedirect.com/science/article/ pii/S1389128613 003472

[36] M. Shafiq, M. Ahmad, A. Irshad, M. Gohar, M. Usman, M. K. Afzal, J. G. Choi, and H. Yu, "Multiple access control for cognitive radiobased ieee 802.11ah networks," Sensors (Switzerland), Vol. 18, No. 7, pp. 1-28, 2018.

[37] A. Gopinath and B. Nithya, "Mathematical and simulation analysis of contention resolution mechanism for ieee 802.11ah networks", Computer Communications, Vol. 124, pp. 87100, 2018. [Online]. Available: https://www.sciencedirect.com/science/article/ pii/S0140366417 308770

[38] S. Taneeru and P. C. Jain, "Performance evaluation of ieee 802.11ah protocol in wireless local area network", Int. Conf. MicroElectronics Telecommun. Eng. ICMETE, p. 578-583, 2016.

[39] Q. T. Ngo, D. N. Minh Dang, Q. Le-Trung, and D. K. Lam, "A novel directional mac in restricted access window for ieee 802.11ah networks", In: Proc. of 26th Int. Conf. Telecommun. ICT, p. 167-171, 2019.

[40] H. Touil and M. Benattou, "Energy-efficient mac protocol based on ieee $802.11 \mathrm{e}$ for wireless multimedia sensor networks", IEEE, 2012. 